\title{
Comparing the Interpretation of Diffusion-Weighted Magnetic Resonance Images Made by Emergency Physicians and On-Call Radiologists
}

\author{
Muhammed Ikbal Sasmaz' (D), Ali Haydar Akca ${ }^{1}$ (D), Ramazan Guven² (D), Burak Altan ${ }^{3}$ (D), Nuri Baraz ${ }^{4}$ (D), Vedat Kirpat ${ }^{5}$ (D), Ali Mahir Gunduz ${ }^{6}$ (D) \\ 'Department of Emergency Medicine, Van Yuzuncu Yil University School of Medicine, Van, Turkey \\ 2Department of Emergency Medicine, Bitlis State Hospital, Bitlis, Turkey \\ ${ }^{3}$ Department of Emergency Medicine, Servergazi State Hospital, Denizli, Turkey \\ ${ }^{4}$ Department of Radiology, Servergazi State Hospital, Denizli, Turkey \\ ${ }^{5}$ Department of Emergency Medicine, Van Training and Research Hospital, Van, Turkey \\ ${ }^{6}$ Department of Radiology, Van Yuzuncu Yil University School of Medicine, Van, Turkey
}

Cite this article as: Sasmaz MI, Akca AH, Guven R, Altan B, Baraz N, Kirpat V, et al. Comparing the Interpretation of Diffusion-Weighted Magnetic Resonance Images Made by Emergency Physicians and On-Call Radiologists. Eurasian J Emerg Med. 2018; 17: 41-4.

\begin{abstract}
Aim: Stroke is a leading cause of adult disability and death worldwide. It is very important to determine the ischemic tissue at risk in stroke patients. Although brain computed tomography (CT) is the first and standard imaging technique, diffusion-weighted magnetic resonance imaging (DW-MRI) is superior to CT and is the optimal imaging technique for the diagnosis of acute ischemic stroke. In our study, we aimed to compare the interpretation of DW-MRI between emergency physicians (EP) and on-call radiologists (OCRs).

Materials and Methods: This multi-centered, prospective study was conducted at three central hospitals from June 1, 2016 to May 31, 2017. DW-MRI images of the patients were first interpreted by the EP and then by the OCR. Finally, DW-MRI images were interpreted by a definitive result team comprised of two radiologists who were blinded to the study. Sensitivity, specificity, positive predictive value, negative predictive value, and $\mathrm{k}$ coefficient were calculated.

Results: In total, 315 patients' DW-MRI images were examined. The interpretation sensitivity and specificity rates of the DW-MRI images for EPs were $95.1 \%$ and $98.7 \%$, respectively, whereas those for OCRs were $98.8 \%$ and $98 \%$, respectively. There was almost perfect agreement ( $\mathrm{K}$ value $>-0.80$ ) regarding DW-MRI interpretations of both OCRs and EPs.

Conclusion: Our study showed that EPs were very successful in interpreting DW-MRI after a short-term training.

Keywords: DW-MRI interpretation, emergency physicians, radiologists

\section{Introduction}

Stroke is one of the most important causes of death and the most common cause of serious physical disability (1). There are two primary types of stroke: ischemic and hemorrhagic. Ischemic strokes are by far the most common, accounting for $87 \%$ of all strokes (2). The possible causes of ischemic stroke are approximately $25 \%$ arteroembolic (large-artery disease), 25\% lacunar (small-vessel disease), $25 \%$ cardioembolic, and $25 \%$ due to other causes (3).

Emergency departments (ED) are the point of access to health care for stroke patients. Thus, timely ED evaluation and diagnosis of stroke are critical (4). It is very important to determine the ischemic tissue at risk in stroke patients. Non-contrast computed tomography (CT) is the first and standard imaging technique for the diagnosis of stroke because it is fast and widely available and generally used to exclude cerebral hemorrhage $(5,6)$. The sensitivity of CT at the onset of cerebral ischemia was $64 \%$ in the European Cooperative Acute Stroke Study (7).
\end{abstract}

ORCID IDs of the authors: M.I.S. 0000-0002-3267-3184; A.A.A. 0000-0001-7372-3582; R.G. 0000-0003-4129-8985; B.A. 0000-0002-63846439; N.B. 0000-0001-9102-5470; V.K. 0000-0001-8168-3058; A.M.G. 0000-0002-4471-4596. 
Diffusion-weighted magnetic resonance imaging (DW-MRI) seems to be superior to $\mathrm{CT}$ and has been described as the optimal imaging technique for the diagnosis and management of acute ischemic stroke (AIS) $(8,9)$. DW-MRI is an advanced technique that is generally used to detect AIS in the ED. The detection rate of brain DW-MRI ischemic lesions in stroke patients is $>95 \%$, whereas it is between 1 in 6 and 2 in 3 in transient ischemic attack (TIA) patients. Speech or motor symptoms, duration of symptoms, and etiology seem to correlate with the rate of DW-MRI positivity (10). The determination of infarct-age is not possible in the first few hours based on DW-MRI characteristics. However, the combined interpretation of DW-MRIimages and the apparent diffusion coefficient (ADC) maps enable the distinction of the duration of infarcts (11).

Because of these advantages, DW-MRI has become a useful and important imaging modality for emergency physicians (EP) especially in subclinical patients, transient ischemic attack (TIA) patients, and those with a history of stroke. The aim of this study was to compare the interpretations made by EP and radiologists on DW-MRI images obtained in the ED for patients with the pre-diagnosis of AIS and to evaluate the adequacy of EP on interpreting the DW-MRI images.

\section{Materials and Methods}

\section{Study design}

This multi-centered, prospective study was carried out at three central hospitals Denizli Servergazi State Hospital, Van Training and Research Hospital, and Bitlis State Hospital. Approval was acquired from the local ethics committee of the University of Health Sciences Van Training and Research Hospital to conduct the study. Patients were selected between 08:00 hours and 17:00 hours on weekdays as radiologists could be contacted more easily. The DW-MRI scans were performed using Siemens, Philips, and Signa G-Explorer 1.5 Tesla MRI machines at the three centers. Trainings on interpreting DW-MRI scans were provided to the EPs by the radiology clinic instructors. These trainings were given as 2 hours for practical and 2 hours for theoretical through a PowerPoint presentation showing diffusion imaging pathologies. Patients were selected during a 1-year period between June 1, 2016, and May 31, 2017. Patients who had stroke symptoms or suspected of stroke and underwent brain DWI were included in the study. The exclusion criteria were patients who were evaluated in other centers and those younger than 18 years.

\section{Image evaluation and data analysis}

Evaluation was performed according to the presence of brain diffusion restriction on DW-MRI (high signal on DW-MRI and low on ADC map). The DW-MRI images of the patients who were admitted to the ED with a suspicion of stroke were first interpreted by the EPs. This preliminary interpretation was noted down by the EP on a study form in the "interpretation of the EP" section. The clinical information of the patient was simultaneously forwarded to the oncall radiologist $(\mathrm{OCR})$ via telephone. The OCR evaluated the images of the patient and forwarded his/her interpretation to the EP. The EP noted down the interpretation of the OCR in the "interpretation of the OCR" section of the study form. Finally, the DW-MRI images were interpreted by the definitive result team comprised of two radiologists who were blinded to the study. All EPs and radiologists in the study were expert doctors.
Table 1. Characteristics of the patients $(n=315)$

\begin{tabular}{|c|c|}
\hline Age (year) & $71.7 \pm 1.2$ \\
\hline \multicolumn{2}{|l|}{$\operatorname{Sex}(\%)$} \\
\hline Male & $166(52.7)$ \\
\hline Female & $149(47.3)$ \\
\hline \multicolumn{2}{|l|}{ Complaints of the patients, $n(\%)$} \\
\hline Right or left side weakness & $78(24.8)$ \\
\hline Speech disorder & $73(23.2)$ \\
\hline Loss of consciousness & $64(20.3)$ \\
\hline Vertigo & $37(11.7)$ \\
\hline Syncope/fainting & $23(7.3)$ \\
\hline Ataxia/gait disturbance & $19(6.0)$ \\
\hline Weakness & $12(3.8)$ \\
\hline Facial paralysis & $9(2.9)$ \\
\hline \multicolumn{2}{|l|}{ Neurological findings of the patients (\%) } \\
\hline Right or left-sided hemiparesis/hemiplegia & $67(21.3)$ \\
\hline Absent & $66(21.0)$ \\
\hline Dysarthria/aphasia & $59(18.7)$ \\
\hline Confusion & $56(17.8)$ \\
\hline Cerebellar dysfunction(s) & $41(13.0)$ \\
\hline Nystagmus & $18(5.7)$ \\
\hline Facial paralysis & $8(2.5)$ \\
\hline \multicolumn{2}{|l|}{ Electrocardiography rhythm, n (\%) } \\
\hline Normal sinus rhythm & $232(72.7)$ \\
\hline Atrial fibrillation & $82(26.0)$ \\
\hline Atrioventricular complete block & $1(0.3)$ \\
\hline
\end{tabular}

\section{Statistical analysis}

Analysis of the data was performed using the Statistical Package for Social Sciences software package 23 (IBM Corporation, Chicago, IL, USA). Specificity, sensitivity, positive predictive value, negative predictive value, positive likelihood ratio, negative likelihood ratio, and $\mathrm{k}$ coefficient were calculated for the interpretations by EP and OCR based on the final report. The inter-rater agreement was graded according to $\mathrm{K}$ values. $\mathrm{A} \mathrm{K}$ value of $0.81-1$ was considered almost perfect agreement, $0.61-0.80$ as substantial agreement, $0.41-0.60$ as moderate agreement, $0.21-0.40$ as fair agreement, and $0-0.20$ as slight agreement. The level of significance was accepted as a p-value of $<0.05$ with $95 \%$ confidence intervals.

\section{Results}

A total of 356 patients who were scheduled to undergo brain DW-MRI were selected for the study. Of these patients, 16 were excluded due to contraindications in MRI, 15 were excluded due to insufficiencies of the preliminary interpretation of the EP or the attending radiologist, and 10 were excluded because they had hemodynamic instability and did not undergo MRI. Finally, 315 patients were included in the study. Of the 315 patients, 132 were from Denizli Servergazi State 
Hospital, 105 were from Van Training and Research Hospital, and 78 were from Bitlis State Hospital. The mean age of the patients was found to be $71.7 \pm 1.22$ years. Of all the patients, 166 (52.7\%) were males and 149 (47.3\%) were females.

The common reasons why the patients presented to the ED were rightor left-sided weakness (24.8\%), speech disorder (23.2\%), and loss of consciousness (20.3\%; Table 1). The common neurological findings of the patients were right- or left-sided hemiparesis/hemiplegia (21.3\%), absent (21\%), and dysarthria/aphasia (18.7\%; Table 1). Overall, 232 (72.7\%) patients had sinus rhythm, $82(26 \%)$ had atrial fibrillation, and $1(0.3 \%)$ had atrioventricular block on electrocardiography; 159 (50.5\%) patients were diagnosed with acute AIS and 36 (11.4\%) were diagnosed with TIA. In total, $120(38.1 \%)$ patients received nonischemic neurological diagnoses or non-neurological diagnoses. Further, $156(49.5 \%)$ patients were hospitalized to the neurology department, $20(6.3 \%)$ were hospitalized to the neurology intensive care unit, and 31 (9.8\%) patients were hospitalized to other clinics with non-neurological diagnoses. Also, 108 (34.3\%) of the 315 patients were discharged from the ED.

Of 315 patients with a suspicion of stroke, 164 (52.1\%) had positive DW-MRI and ADC maps findings. The DW-MRI interpretation sensitivity and specificity levels for the EPs were $95.1 \%$ and $98.7 \%$, respectively, as against $98.8 \%$ and $98 \%$, respectively, for the OCR. According to the final report, there was an almost perfect agreement regarding DW-MRI interpretations of both OCR and EP ( $\mathrm{K}$ value; 0.968 and 0.936 , respectively). Data on brain DW-MRI interpretation of EP and OCR are shown in Table 2.

\section{Discussion}

With the current prolongation in the expected lifespan of people, the number of patients with stroke has also increased. Since the ED is the access point to healthcare for critical patients, such as stroke patients, they constitute the most important step in the early diagnosis and treatment of stroke (4). In the presence of findings of acute stroke, the most commonly admitted imaging method is brain CT. CT is important particularly in the diagnosis or exclusion of hemorrhagic stroke. However, its diagnostic sensitivity is low in ischemic stroke, which forms the majority of the cases with stroke in the short term (7).

DW-MRI has become the mostly preferred imaging method since it is the most sensitive and specific method in the diagnosis of ischemic stroke, it can be accessed easily in EDs and is time saving. However, DW-MRI has been interpreted together with ADC maps. By this means, acute infarction may easily be diagnosed, and the discrimination of acute infarction from chronic infarction can be made. Especially in patients with subclinical properties, those with altered consciousness, in the elderly patients for whom physical examination is difficult, and in those with suspicious TIA, DW-MRI helps the EP in the diagnosis or exclusion of AIS $(8,9)$.

The interpretation of the images obtained in different imaging techniques in the ED is generally provided by radiologists. Currently, images are easily directed to radiologists via professional methods, suchaspicturearchivingand communicationssystem; communication methods, such as cellular phones; or applications such as WhatsApp ${ }^{\circledR}$ (12). However, for critical patients where early diagnosis is crucial, communication to the radiologists may not always be possible. Although Hunter and colleagues (13) suggest that radiologic imaging in the ED should only be interpreted by radiologists, Torreggiani et al. (14) stated that simultaneous radiologist interpretation was not possible in a short time in many institutions. Therefore, EPs complain about the inadequacy of the current reporting system. Thus, the correct interpretation of radiological images, such as CT or MRI, by EP in critical patients is extremely important in the diagnosis and management of these patients. EPs with that knowledge progress with regard to interpreting radiological images.

There are several studies comparing EPs and radiologists in interpreting radiological images. Majority of these studies were used to be on ultrasonography and x-ray at the beginning $(15,16)$. However, studies comparing interpretations on CT images have gained importance recently $(17,18)$. With the advantage of being informed about the history, physical examination, and clinical findings of the patients in most of the studies, the success of EPs in interpreting radiological images was observed to be higher. In the study of Kartal et al. (19) on the CT images obtained from patients with trauma, the interpretations of EPs were found to be very successful especially for the thoracic and brain CT interpretations and moderately successful in abdominal and vertebral CT interpretations. In the study of Hochhegger et al. (17), fair and moderate agreements (0.39 and 0.42) were demonstrated between EP and radiologists in interpreting CT pulmonary angiography in the diagnosis of pulmonary embolism. Oray et al. found an acceptable level of agreement for the interpretation of DW-MRI among four EPs in a retrospective study (20). However, the number of patients included in the study was low. To our knowledge, there is not prospective study that investigated the compatibility of EP and OCR in interpreting the images of DW-MRI, which has been admitted frequently today; our study was the first in literature on the subject.

The adequacy of EP on interpreting brain DW-MRI images were found to be similar to those of OCR in our study (K value: $0.936-0.968$ ) and highly compatible according to precise interpretations of two independent radiologists, which we accept as the gold standard ('very good agreement'). Among the patients, 159 were diagnosed to be AIS and 36 were TIA, whereas 120 were diagnosed to have different diseases. In the management of these patients, EP have either confirmed their pre-diagnoses with the help of DW-MRI, or excluded AIS from the differential diagnosis.

\section{Study limitations}

Some of the patients included in the study were hospitalized by the neurologists based on the clinical findings and DW-MRI interpretations of OCR. Some were hospitalized and discharged from different units with non-neurological diagnoses. No future follow-up was planned for these patients, and no further on images were maintained. Therefore, a possibility of misdiagnosis or misinterpretation of radiological images is a limitation of this study. In addition, the EPs were more advantageous than radiologists in interpreting because they were not blinded to the CT results and clinic information of the patients. 


\section{Conclusion}

The high compliance observed in the interpretations of DW-MRI by EPs to those of radiologists for AIS was significant and satisfactory.

Ethics Committee Approval: Ethics committee approval was received for this study from the ethics committee of University of Health Sciences Van Training and Research Hospital.

Informed Consent: Written informed consent was not needed because patients in the study had not been applied any interference and normal procedure was being processed. Also patients' images were evaluated based on the file number.

Peer-review: Externally peer-reviewed.

Author Contributions: Concept - M.I.S.; Design - M.I.S.; Supervision - M.I.S., A.H.A., R.G.; Resources - M.I.S., V.K., R.G.; Materials - M.I.S., B.A.; Data Collection and/or Processing - M.I.S., A.H.A., R.G., V.K.; Analysis and/or Interpretation - M.I.S., A.H.A., B.A., N.B., V.K., A.M.G.; Literature Search - M.I.S., V.K.; Writing Manuscript - M.I.S., A.H.A.; Critical Review - M.I.S.; Other - V.K., R.G., A.M.G., N.B.

Acknowledgements: I would like to express myspecial appreciation and thanks to doctors and healthcare professionals who participated in this study.

Conflict of Interest: The authors have no conflict of interest to declare.

Financial Disclosure: The authors declared that this study has received no financial support.

\section{References}

1. Feigin VL, Forouzanfar $M H$, Krishnamurthi $R$, Mensah GA, Connor $M$, Bennett DA, et al. Global and regional burden of stroke during 19902010: findings from the global burden of disease study 2010. Lancet. 2014; 383: 245-55. [CrossRef]

2. Mozaffarian D, Benjamin EJ, Go AS, Arnett DK, Blaha MJ, Cushman M, et al. Heart disease and stroke statistics-2015 update: a report from the American Heart Association. Circulation. 2015; 131: e29-e322.

3. Ay H, Furie KL, Singhal A, Smith WS, Sorensen AG, Koroshetz WJ. An evidence-based causative classification system for acute ischemic stroke. Ann Neurol. 2005; 58: 688-97. [CrossRef]

4. Hacke W, Donnan G, Fieschi C, Kaste M, von Kummer R, Broderick JP, et al. ATLANTIS Trials Investigators; ECASS Trials Investigators; NINDS rt-PA Study Group Investigators. Association of outcome with early stroke treatment: pooled analysis of ATLANTIS, ECASS, and NINDS rt-PA stroke trials. Lancet. 2004; 363: 768-74. [CrossRef]

5. Kumar G, Goyal MK, Sahota PK, Jain R. Penumbra, the basis of neuroimaging in acute stroke treatment: Current evidence. J Neurol Sci. 2010; 288: 13-24. [CrossRef]
6. Barber PA, Hill MD, Eliasziw M, Demchuk AM, Pexman JH, Hudon ME, et al. Imaging of the brain in acute ischaemic stroke: comparison of computed tomography and magnetic resonance diffusion-weighted imaging. J Neurol Neurosurg Psychiatry. 2005; 76: 1528-33. [CrossRef]

7. Von Kummer R, Bourquain H, Bastianello S, Bozzao L, Manelfe C, Meier D, et al, for the European Cooperative Acute Stroke Study II Group. Early prediction of irreversible brain damage after ischemic stroke at CT. Radiology. 2001; 219: 95-100. [CrossRef]

8. Ledezma CJ, Fiebach JB, Wintermark M. Modern imaging of the infarct core and the ischemic penumbra in acute stroke patients: CT versus MRI. Expert Rev Cardiovasc Ther. 2009; 7: 395-403. [CrossRef]

9. Merino JG, Warach S. Imaging of acute stroke. Nat Rev Neurol. 2010; 6: 560-71. [CrossRef]

10. Provenzale JM, Sorensen AG. Diffusion-weighted MR imaging in acute stroke: theoretic considerations and clinical applications. AJR Am J Roentgenol. 1999; 173: 1459-67. [CrossRef]

11. Gass A, Niendorf T, Hirsch JG. Acute and chronic changes of the apparent diffusion coefficient in neurological disorders - biophysical mechanisms and possible underlying histopathology. J Neurol Sci. 2001; 186(Suppl 1): S15-S23.

12. Sistrom CL, Dreyer K, Weilburg JB, Perloff JN, Tompkins CP, Ferris TG. Images of imaging: how to process and display imaging utilization for large populations. AJR Am J Roentgenol. 2015; 204: W405-20.

13. Hunter TB, Krupinski EA, Hunt KR, Erly WK. Emergency department coverage by academic department of radiology. J Acad Radiol. 2000; 7 : 165-70. [CrossRef]

14. Torreggiani WC, Nicolaou S, Lyburn ID, Harris AC, Buckley AR. Emergency radiology in Canada: a national survey. Can Assoc Radiol J. 2002; 53: 160-7.

15. Summers SM, Scruggs W, Menchine MD, Lahham S, Anderson C, Amr $\mathrm{O}$, et al. A prospective evaluation of emergency department bedside ultrasonography for the detection of acute cholecystitis. Ann Emerg Med. 2010; 56: 114-22. [CrossRef]

16. Fox JC, Solley M, Anderson CL, Zlidenny A, Lahham S, Maasumi K Prospective evaluation of emergency physician performed bedside ultrasound to detect acute appendicitis. Eur J Emerg Med. 2008; 15: 80-5. [CrossRef]

17. Hochhegger B, Alves GR, Chaves M, Moreira AL, Kist R, Watte G, et al. Interobserver agreement between radiologists and radiology residents and emergency physicians in the detection of PE using CTPA. Clin Imaging. 2014; 38: 445-7. [CrossRef]

18. Klein EJ, Koenig M, Diekema DS, Winters W. Discordant radiograph interpretation between emergency physicians and radiologists in a pediatric emergency department. Pediatr Emerg Care. 1999; 15: 245-8. [CrossRef]

19. Kartal ZA, Kozaci N, Cekic B, Beydilli I, Akcimen M, Guven DS, et al. CT interpretations inmultiply injured patients: comparison of emergency physicians and on-call radiologists. Am J Emerg Med .2016; 34: 2331-5. [CrossRef]

20. Oray D, Limon O, Ertan C, Aydinoglu Ugurhan A, Sahin E. Inter-Observer Agreement on Diffusion-Weighted Magnetic Resonance Imaging Interpretation for Diagnosis of Acute Ischemic Stroke Among Emergency Physicians. Turk J Emerg Med. 2015; 15: 64-8. [CrossRef] 